\title{
Scale nucleation natural precursors: a case study of "micro/nanodust" impurities nature in laboratory aqueous samples obtained from Moscow tap water
}

\author{
K. Popov, ${ }^{1 *}$ M. Oshchepkov, ${ }^{1,2}$ A. Kovalenko, ${ }^{1,2}$ A. Redchuk, ${ }^{1}$ J. Dikareva ${ }^{1}$ \\ and I. Pochitalkina ${ }^{2}$ \\ ${ }^{1}$ JSC "Fine Chemicals R\&D Centre”, Krasnobogatyrskaya str. 42, b1, Moscow, 107258, \\ Russian Federation \\ ${ }^{2}$ D. Mendeleev University of Chemical Technology of Russia, Miusskaya sq. 9, 125047 \\ Moscow, Russian Federation \\ *E-mail:ki-popov49@yandex.ru
}

\begin{abstract}
Recent studies revealed that the bulk scale nucleation has a heterogeneous character with nano/microdust impurities acting as scale templates in aqueous medium. Thus, the scale inhibition phenomenon is associated exactly with nano/microdust surface blockage by antiscalant molecules. Meanwhile, little is known about the nature of dust impurities. Therefore, the present report is focused solely on the study of suspended solid matter occurring in reagent grade water and in some tap water samples by means of particle counter technique, ICP analysis, DLS, SEM and X-ray diffraction. A particle counter was used to demonstrate that foreign solid particles sized over $100 \mathrm{~nm}$ are inevitably present in any reagent grade chemicals in amounts from $10^{2}$ to $\geq 10^{5}$ units per $1 \mathrm{ml}$ (from $10^{5}$ to $\geq 10^{8}$ per $\mathrm{dm}^{3}$ ), including water for ion chromatography (Sigma-Aldrich), $\mathrm{KCl}$ (Sigma-Aldrich) and $\mathrm{HNO}_{3}$ for microelectronics (Avantor Performance Materials Ltd). At the same time, DLS revealed for $\mathrm{HNO}_{3}$ that the microfraction $(\geq 100 \mathrm{~nm}$ ) registered by a particle counter constitutes less than several per cent of the solid impurities sized below $100 \mathrm{~nm}$. It was found that a common laboratory practice of dust removal via 200 or $220 \mathrm{~nm}$ filters provides a substantial decrease in the concentration of microparticles but only slightly affects the fraction below $200 \mathrm{~nm}$, to say nothing of nanoparticles $(1 \mathrm{~nm} \leq$ size $\leq 100 \mathrm{~nm})$. Moreover, this operation does not provide even complete removal of the fraction sized over $200 \mathrm{~nm}$. Meanwhile, deionized water demonstrates a significantly lower concentration of solid impurities larger than $100 \mathrm{~nm}(320$ per $1 \mathrm{ml})$ than distilled water (4300 per $1 \mathrm{ml}$ ). A combination of ICP, SEM and X-Ray diffraction analyses of distilled water produced from Moscow tap water indicated that the elemental composition of microparticles includes $\mathrm{Si}, \mathrm{O}, \mathrm{Ca}, \mathrm{Fe}$, and $\mathrm{Al}$. A comparison of microparticle (size $>100 \mathrm{~nm}$ ) content in tap water samples taken in different cities worldwide demonstrates that there is no significant difference between them. The particle concentration ranges from 280000 (Amsterdam) to 440000 (Rome) units per $1 \mathrm{ml}$ which is almost within the $20 \%$ measurement error. Thus, the problem of micro/nanodust occurrence in distilled/deionized water is common for many laboratories worldwide involved in water treatment studies.
\end{abstract}


Keywords: water, nucleation, scale formation, nano/micro impurities, particle counter, SEM, DLS, "Micro/Nanodust" removal.

Received: August 28, 2020. Published: September 17, 2020

doi: $\underline{10.17675 / 2305-6894-2020-9-3-20}$

\section{Introduction}

Nucleation is the first step of any industrial scale formation process [1-4]. Although many classical and nonclassical nucleation theories have been developed, nucleation mechanism continues to be one of the most poorly understood and disputable phenomena over the past half century [1,2]. Meanwhile, this step is of key importance for scale inhibition process understanding as far as the threshold effect of antiscalants is attributed exactly to the nucleation phase of scaling $[5,6]$.

Generally, the bulk crystallization in an aqueous supersaturated solution of a sparingly soluble salt may pass two different routes of initial nucleation: homogeneous and heterogeneous [3]. The former mechanism assumes spontaneous aggregation of corresponding ions, while the latter one suggests, that nucleation takes place at solid colloidal impurities (nano/microdust), occasionally present in aqueous medium. These impurities are expected to serve as crystallization centers [7,8]. Despite the fact that the heterogeneous scenario in the bulk is energetically more favorable than the homogeneous one, the latter pathway is broadly accepted and dominates in water treatment science [9-17]. However, recent results of Gromov [18] and of our research group [19-21] clearly indicate that (i) the dominant scale formation mechanism in a bulk aqueous solution has a heterogeneous character with foreign solid impurities acting as nucleation centers, and (ii) scale inhibition phenomenon is associated exactly with nano/microdust surface blockage by antiscalant molecules. Thus, an antiscalant-nanodust interaction becomes of key importance in water treatment science.

Meanwhile, little is known on the nanodust nature so far. There are just a few reports on nano/microdust present in natural waters [7,8] and in ultrapure chemicals [22-24]. Unfortunately, the chemical composition of uncontrolled solid matter impurities is poorly described there. Thus, present paper represents a case study of solid impurities in distilled water and in deionized water obtained from Moscow municipal water supply system and then used in everyday laboratory practice [19-21], particularly in scale inhibition experiments.

\section{Experimental}

\subsection{Reagents}

Two types of water were studied: in-house distilled and in-house deionized one. The distilled water was obtained via common distillation procedure operating a common industrial distiller (DE-4 TZMOI, Russia), and municipal Moscow tap water. This distillate is further denoted as distilled water (DI water). It was analyzed for $\mathrm{Al}, \mathrm{Fe}, \mathrm{Ca}, \mathrm{Mg}, \mathrm{Si}, \mathrm{Zn}, \mathrm{K}$ and $\mathrm{Na}$ 
content by ICP, and for solid particles content by a particle counter. Then the DI water $(100 \mathrm{ml})$ was filtered manually through $0.2 \mu \mathrm{M}$ hydrophilic polytetrafluorethylene (PTFE) Millipore Millex-LG filter. The filtered DI water was re-tested for microelements and particles content. The differences in microelement concentrations were assigned to the solid particles. Millipore Millex-LG filter was then dried for 7 days at ambient temperature, dismantled and analyzed by SEM. A virgin Millipore Millex-LG filter was used as a blank reference sample.

The deionized water was obtained from the DI water, which passed an ion exchange procedure, followed by microfiltration (Simplicity UV, "Merck Millipore SAS"). This one served as a reference for DI water. Another water reference sample was obtained from Sigma-Aldrich (Water for ion chromatography, Cat. \#00612, Lot BCCC3484) and used without any further treatment.

Besides, several tap water samples from different European and US municipalities were taken during a summer season of 2019. They were analyzed for solid impurities content by particle counter technique for comparison with the Moscow tap water.

In addition, the $70 \%$ nitric acid (Avantor Performance Materials Ltd.) for semiconductors with limited suspended microparticle content, and a solid sample of high purity $\mathrm{KCl}$ (99.0-100.5\%); CAS 7447-40-7; Sigma-Aldrich) were used as reference compounds. The latter was used as $0.1 \mathrm{~mol} \cdot \mathrm{dm}^{-3}$ solution in an in-house deionized water.

All experiments were run in a certified laboratory room (ISO 14644-1, class 8) with a limited dust microparticle air phase content, controlled by an air particle counter Particle Measuring Systems Inc. with analytical channels $0.3 ; 0.5 ; 5.0 \mu \mathrm{m}$.

\subsection{ICP analysis}

Element analysis of water samples was performed operating iCAP 6300 Duo (Thermo Scientific) instrument. Analysis was performed for 7 elements. The results were compared with the Sigma-Aldrich Catalogue Sigma-Aldrich water sample (Water for ion chromatography, Cat. \#00612, lot BCCC3484).

\subsection{SEM analysis}

Solid impurities morphology and chemical composition were examined with scanning electron microscope (SEM), Hitachi TM3030, coupled with energy dispersive X-Ray spectroscopy (EDS) analysis unit Quantax 70. The sample examinations by SEM were performed using $15 \mathrm{kV}$ accelerating voltage in Charge-Up Reduction Mode; crystal phase was located on filter and the working distance was $4.1 \mathrm{~mm}$.

\subsection{Particle counter analysis}

Particle counter was used for quantitative characterization of suspended solid impurities concentration of the particles sized $\geq 100 \mathrm{~nm}$. The concentration and size of solid particles in water samples were measured by a particle counter SLS-1100 (Particle Measuring 
Systems Inc.) This instrument provides a particle concentration measurement within the size range from $100 \mathrm{~nm}$ to $1 \mathrm{~mm}$.

\subsection{X-Ray crystal characterization}

An attempt was made to use X-Ray diffraction analysis (XRD) for suspended solid matter characterization that was retarded on the filters. In order to reach the goal amounts of 5 liter of water or $50 \mathrm{ml}$ of aqueous solution of particular chemical was subjected to pass through $0.2 \mu \mathrm{m}$ Hydrophilic polytetrafluorethylene (PTFE) Millipore Millex-LG filter with an area of $5 \mathrm{~cm}^{2}$. The pieces of dried filter with some solids detained by its surface have been analyzed with Bruker D8 Advance diffractometer (Cu KD; Ni-filter; LYNXEYE detector). The XRD phase identification was done with Joint Committee on Powder Diffraction Standards (JCPDS) database, and relative phase content was estimated with Topaz R software (Bruker AXS). However, the total amount of deposited particles appeared to be not sufficient to provide the diffractograms of the required quality. Thus, these data are not considered further.

\subsection{Dynamic light scattering}

Liquid phase was monitored by the dynamic light scattering technique (DLS) for qualitative characterization of suspended solid impurities within the particles range from $1 \mathrm{~nm}$ to $1000 \mathrm{~nm}$. DLS experiments were performed at $25^{\circ} \mathrm{C}$ with Malvern Nano ZS instrument $(\lambda=633 \mathrm{~nm}$, operating power $4 \mathrm{~mW})$ at $\Theta=173^{\circ}$.

\section{Results and Discussion}

Based on the logical assumption that a true complete characterization of suspended solid matter in reagent grade chemicals is hardly possible due to a broad variation of such impurities chemical composition, our study is limited to the water, used by our research group in scale formation studies. Indeed, chemical composition of such solid impurities depends in general on manufacturer, purification technique etc., as well as on the difficulties of their characterization against the background of water-soluble impurities. Although the emphasis is done on distilled water and on deionized water, derived from Moscow municipal tap water, the results might be of interest for researchers from around the globe. At the same time, this supplement study is intended to put some additional light on our previous and future results in scale inhibition. We understand that our present study embraces mostly a larger fraction of the particles present (sized $>100 \mathrm{~nm}$ ). Even though, it is believed to become a particular step forward in our knowledge of solid matter impurities, present on $\mathrm{ppm} / \mathrm{ppb}$ level in chemicals.

\subsection{Aqueous phase characterization by ICP, DLS and particle counter technique}

The ICP data, presented in Table 1, indicate that both distilled and deionized water prepared from Moscow tap water, and then used in the present study, fit the standards of pure 
chemicals [25]. Actually, both of them have the same content of microelement impurities, as the water sample, provided by Sigma-Aldrich.

The differences in $\mathrm{Al}, \mathrm{Fe}$ and $\mathrm{Ca}$ concentration between the distilled water samples before and after filtration, demonstrate that these elements are partly (Al, $\mathrm{Fe}$ ) or completely (Ca) present in the form of solids, retarded by the filter material, unlike $\mathrm{K}$ and $\mathrm{Na}$, which exist completely as water soluble species $\left(\mathrm{Na}^{+}, \mathrm{K}^{+}\right)$. At the same time, residual $\mathrm{Al}$ and $\mathrm{Fe}$ content in the filtered DI water cannot be definitely assigned either to solid, or to aqueous phase. Both forms are possible. According to Table 1, Al and Fe may penetrate the filter material as nanosolids with sizes below $200 \mathrm{~nm}$, as well as in the form of ions. Tentatively the nature of solid impurities in distilled water can be assigned to $\mathrm{Al} / \mathrm{Fe}$ hydroxo/oxides and to calcium carbonate (Ca). Notably, an increase in $\mathrm{Na}$ and $\mathrm{Zn}$ content in filtrate relative to initial DI water (Table 1) demonstrates that the membrane material itself is capable to emit some impurities. Si was not detected by ICP analysis. However, it was found on filter surfaces after water filtration through $0.2 \mu \mathrm{m}$ Millipore Millex-LG filter.

Solid state characteristics of impurities in analytical grade laboratory chemicals are usually overlooked, mainly because they are present in low concentrations. Thus, it is a challenge for a researcher. The results presented in Tables 2 and 3 reveal clearly that microparticles with a size over $100 \mathrm{~nm}$ are inevitably present in any high purity aqueous sample in significant amounts ranging from $10^{2}$ to $10^{5}$ units in $1 \mathrm{ml}$, or from $10^{5}$ to $10^{8}$ in $1 \mathrm{dm}^{3}$. Notably, the distilled water produced in our laboratory has the solid contaminants of the same order of magnitude as the Sigma-Aldrich sample, while our deionized water contains such impurities in a 10-fold smaller amount.

Table 1. Water samples characterization by ICP.

Element content, ppm

\begin{tabular}{|c|c|c|c|c|c|}
\hline \multirow{2}{*}{ Element } & \multirow{2}{*}{ Distilled water } & \multirow{2}{*}{$\begin{array}{l}\text { Distilled water } \\
\text { after } 200 \mathrm{~nm} \\
\text { filter treatment }\end{array}$} & \multirow{2}{*}{ Deionized water } & \multicolumn{2}{|c|}{ Sigma-Aldrich water } \\
\hline & & & & Catalo & Found \\
\hline $\mathrm{Al}$ & $0.0047 \pm 0.0005$ & $0.0040 \pm 0.0004$ & 0.001 & $\leq 0.005$ & 0.0010 \\
\hline $\mathrm{Fe}$ & $0.0018 \pm 0.0002$ & $0.0010 \pm 0.0002$ & $0.0013 \pm 0.0001$ & $\leq 0.005$ & $0.0011 \pm 0.0001$ \\
\hline $\mathrm{Ca}$ & $0.0006 \pm 0.0001$ & Not found & Not found & $\leq 0.01$ & $0.0005 \pm 0.0001$ \\
\hline $\mathrm{Na}$ & $0.0007 \pm 0.0002$ & $0.0024 \pm 0.0002$ & Not found & $\leq 0.01$ & $0.0005 \pm 0.0001$ \\
\hline K & $0.0017 \pm 0.0003$ & $0.0017 \pm 0.0002$ & Not found & $\leq 0.01$ & $0.0026 \pm 0.0002$ \\
\hline $\mathrm{Zn}$ & $0.0007 \pm 0.0001$ & $0.0012 \pm 0.0001$ & $0.0005 \pm 0.0001$ & $\leq 0.005$ & $0.0005 \pm 0.0001$ \\
\hline $\mathrm{Si}$ & Not detected & Not detected & Not detected & Not specified & $0.0005 \pm 0.0004$ \\
\hline
\end{tabular}

Unfortunately, most of commercial particle counters do not cover the nano-range $(1 \mathrm{~nm} \leq$ size $\leq 100 \mathrm{~nm})$ providing $30 \mathrm{~nm} \leq$ size $\leq 100 \mathrm{~nm}$ at the best [26]. However, an 
extrapolation of data, presented in Table 2 indicates clearly that the number of nanoparticles in $1 \mathrm{ml}$ exceeds substantially that one of microparticles. At the same time, it should be noted that all the background solid suspended particles, listed in Tables 2 and 3 correspond to the $\mathrm{ppb}$ level, e.g., their presence fits qualification "reagent grade". The data presented in Tables 2, 3 demonstrate that filtration with $200 \mathrm{~nm}$ filter reduces the total number of solid microimpurities from two-fold (DI water) to hundred-fold ( $\mathrm{KCl}$ solution), but it fails to provide a complete foreign particles sized larger than $200 \mathrm{~nm}$ removal, to say nothing of the fractions sized between 1 and $200 \mathrm{~nm}$.

Table 2. Water samples characterization by a particle counter.

\begin{tabular}{|c|c|c|c|c|c|}
\hline \multirow{2}{*}{ Sample } & \multirow{2}{*}{$\mathbf{p H}$} & \multicolumn{4}{|c|}{ Cumulative number of foreign particles in $1 \mathrm{ml}$} \\
\hline & & $\geq 100 \mathrm{~nm}$ & $\geq 200 \mathrm{~nm}$ & $\geq 300 \mathrm{~nm}$ & $\geq 500 \mathrm{~nm}$ \\
\hline In-house distilled water; $3 \mu \mathrm{S} / \mathrm{cm}$ & 5.65 & $4300 \pm 40$ & $1390 \pm 20$ & $640 \pm 10$ & $236 \pm 9$ \\
\hline $\begin{array}{l}\text { In-house distilled water after filtration; } \\
\qquad 200 \mathrm{~nm}, 4 \mu \mathrm{S} / \mathrm{cm}\end{array}$ & 5.80 & $2490 \pm 70$ & $130 \pm 10$ & $53 \pm 8$ & $22 \pm 4$ \\
\hline In-house deionized water; $0.056 \mu \mathrm{S} / \mathrm{cm}$ & 6.30 & $320 \pm 10$ & $60 \pm 10$ & $23 \pm 8$ & $9 \pm 3$ \\
\hline Water, Sigma-Aldrich, $1.8 \mu \mathrm{S} / \mathrm{cm}^{*}$ & 5.60 & $3040 \pm 20$ & $630 \pm 10$ & $170 \pm 20$ & $130 \pm 10$ \\
\hline
\end{tabular}

*The solid particles content is not specified.

Anyhow the data for $0.1 \mathrm{~mol} \cdot \mathrm{dm}^{-3} \mathrm{KCl}$ solution in deionized water, and for the same sample after filtration with $200 \mathrm{~nm}$ filter (Table 3) indicate, that ultrapure water and ultrapure chemical from Sigma-Aldrich do not guarantee absence of suspended particles in a sample. In these relevance the numerous reports on homogeneous crystallization of gypsum and calcium carbonate, studied without a proper nanodust control [12-17] look very arbitrary. Moreover, even those reagents for semiconductors that are specially focused on a maximal decrease of solid impurities (nitric acid, Table 3) still contain such particles in sufficient amounts. Thus, it is obvious, that any ultrapure aqueous solution always contains foreign nano- and micro-particles.

Notably, the dissolution of high purity $\mathrm{KCl}$ in deionized water results in a dramatic increase of microparticle concentration relative to deionized water, Table 3. This high particle content is sufficiently diminished by filtration $(200 \mathrm{~nm})$. However, such a filtration does not compensate initial increase: the residual particle concentration in 0.1 molar $\mathrm{KCl}$ solution still remains 5-fold higher than that one for deionized water.

On the other hand, the differences in microelements concentration (ICP) between DI water, filtrated DI water and deionized water being compared with corresponding particle concentration (particle counter) give some space for the microdust large particles fraction chemical characterization. 
Table 3. Nitric acid and $\mathrm{KCl}$ aqueous solution samples characterization by a particle counter.

\begin{tabular}{cccccc}
\hline Sample & $\mathbf{p H}$ & \multicolumn{3}{c}{ Cumulative number of foreign particles in 1 $\mathbf{~ m l}$} \\
\cline { 3 - 6 } & & $\mathbf{2 1 0 0} \mathbf{~ n m}$ & $\mathbf{2 0 0} \mathbf{~ n m}$ & $\mathbf{2 3 0 0} \mathbf{~ n m}$ & $\mathbf{2 5 0 0} \mathbf{~ n m}$ \\
\hline $\begin{array}{c}70 \% \text { Nitric acid (Avantor } \\
\text { Performance Materials Ltd.) }\end{array}$ & $<1$ & $4700 \pm 80$ & $300 \pm 10$ & $40 \pm 8$ & $30 \pm 5$ \\
\hline $\begin{array}{c}0.1 \mathrm{~mol} \cdot \mathrm{dm}^{-3} \mathrm{KCl} \\
\text { in deionized water }\end{array}$ & 6.25 & $268000 \pm 2000$ & $32000 \pm 800$ & $14000 \pm 600$ & $7000 \pm 90$ \\
\hline $\begin{array}{c}0.1 \mathrm{~mol} \cdot \mathrm{dm}^{-3} \mathrm{KCl} \\
\text { in deionized water after } \\
\text { filtration; } 200 \mathrm{~nm}\end{array}$ & 6.05 & $1500 \pm 50$ & $24 \pm 6$ & $10 \pm 4$ & $6 \pm 3$ \\
\hline
\end{tabular}

DLS analysis fails to detect suspended solids in deionized water, in distilled water, and in filtrated $\mathrm{KCl}$ aqueous solution due to their low concentration (below 5000 particle counter detectable units in $1 \mathrm{ml}$ ). Meanwhile, in nitric acid with similar particle concentration (4700 particle counter detectable units in $1 \mathrm{ml}$ ) and in non-filtered $\mathrm{KCl}$ solution (268 000 particle counter detectable units in $1 \mathrm{ml}$ ) some unstable light scattering bands are detected, Figure 1.
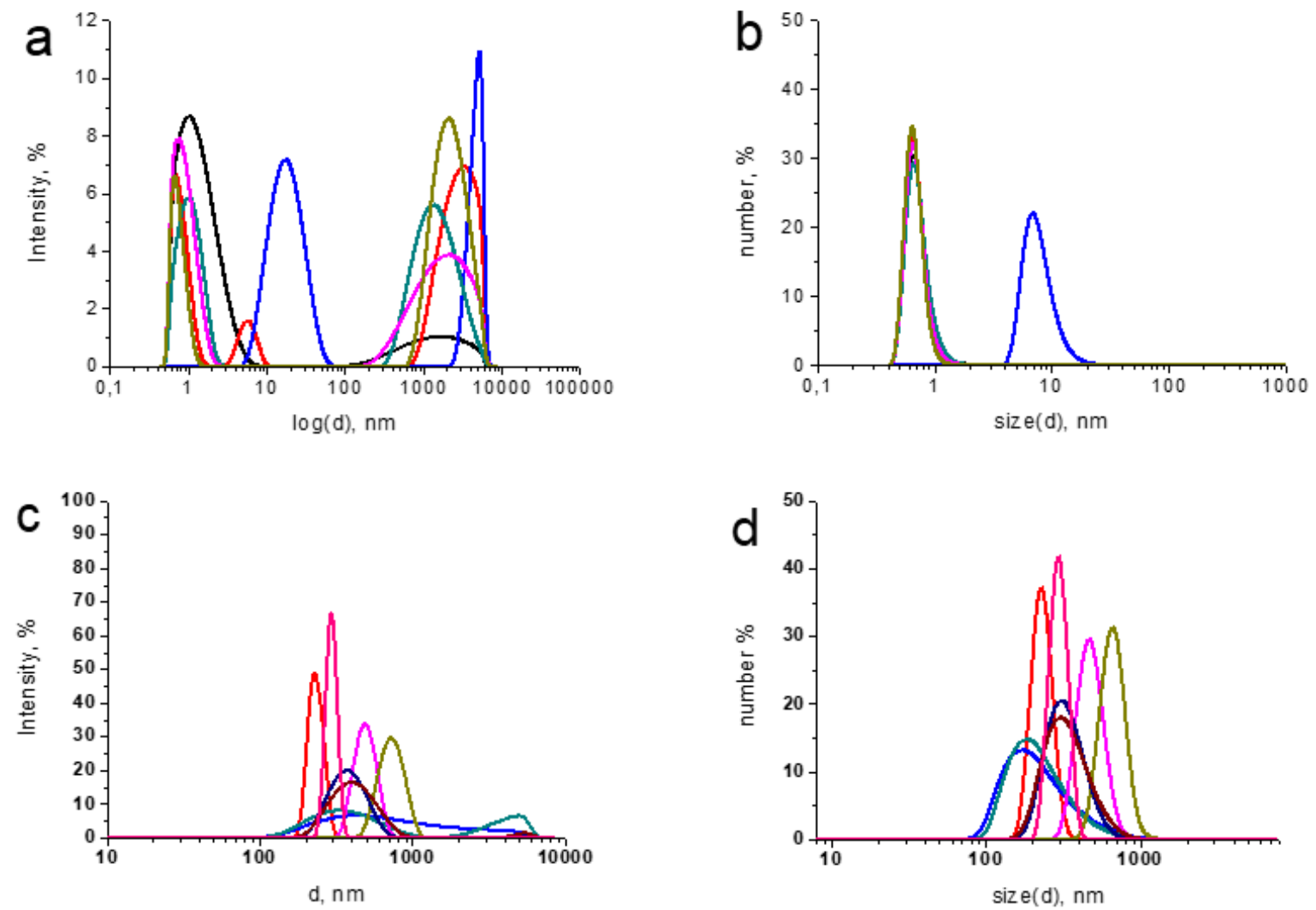

Figure 1. Sequential replicates of DLS particle size distribution by intensity (a,c) and by number $(b, d)$ for nitric acid $(\mathrm{a}, \mathrm{b})$, and $\mathrm{KCl}$ solution in deionized water $(\mathrm{c}, \mathrm{d})$. 
Two different particle size distribution modes have been used: by intensity, and by number. The former is more sensitive to the fine particles, while the latter one-to the larger ones. Here, each band corresponds to a certain replicate measurement. For the nitric acid (Figure 1a) an intensity distribution reveals presence of both nanoparticle fraction (size below $100 \mathrm{~nm}$ ) and microparticle fraction (size above $100 \mathrm{~nm}$ ). The latter one corresponds to those particles that are already detected by a particle counter, while the former one indicates also presence of those particles, which are beyond the particle counter sensitivity. A poor reproducibility of size distribution arises from the low particles concentration: each moment a spontaneously formed combination of solids within the laser beam is changing. This effect is negligible for high particle concentration, but it becomes critical for the low particle content. Thus, the bands are "wandering" within a certain sizes range. Anyhow, these data reflect correctly the presence of both nano- and microfractions of solid impurities in nitric acid.

Table 4. Cumulative number of suspended particles (thousands in $1 \mathrm{ml}$ ) in a municipal tap water measurement channel.

\begin{tabular}{cccccc}
\hline \multirow{2}{*}{$\begin{array}{c}\text { Measurement } \\
\text { channel, nm }\end{array}$} & $\begin{array}{c}\text { Obzor City, } \\
\text { Bulgaria }\end{array}$ & $\begin{array}{c}\text { Straubing, } \\
\text { Germany }\end{array}$ & $\begin{array}{c}\text { Moscow, } \\
\text { Russia }\end{array}$ & $\begin{array}{c}\text { City of Playa } \\
\text { Paraiso, Spain }\end{array}$ & $\begin{array}{c}\text { Pula City, } \\
\text { Croatia }\end{array}$ \\
\hline$>100$ & 420 & 420 & 300 & 320 & 420 \\
$>200$ & 120 & 93 & 53 & 250 & 240 \\
$>300$ & 38 & 28 & 14 & 146 & 100 \\
$>500$ & 15 & 11 & 6 & 102 & 47 \\
\hline & Rimini, & Amsterdam, & San Francisco, & Rome, & Nice, \\
& Italy & Netherlands & USA & Italy & France \\
\hline 100 & 300 & 280 & 366 & 437 & 380 \\
$>200$ & 160 & 110 & 140 & 140 & 230 \\
$>300$ & 53 & 36 & 52 & 38 & 110 \\
\hline 500 & 24 & 16 & 25 & 15 & 57 \\
\hline
\end{tabular}

$* \pm 20 \%$

At the same time, the distribution by number (Figure 1b) demonstrates that nanoparticle fraction dominates microparticles. In this mode, only nanofraction remains visible, indicating at least $10^{6}$-fold excess of nanoparticles number over that one of microparticles. Indeed, according to Rayleigh's law, the light scattering intensity increases roughly in proportion to $R^{6}$, where $R$ corresponds to a particle radius [27,28], when $R \leq \lambda / 15$. Thus, even traces of larger particles would be visible in the background of dominating small ones in the intensity mode, while in distribution by number they will be lost in noise. 

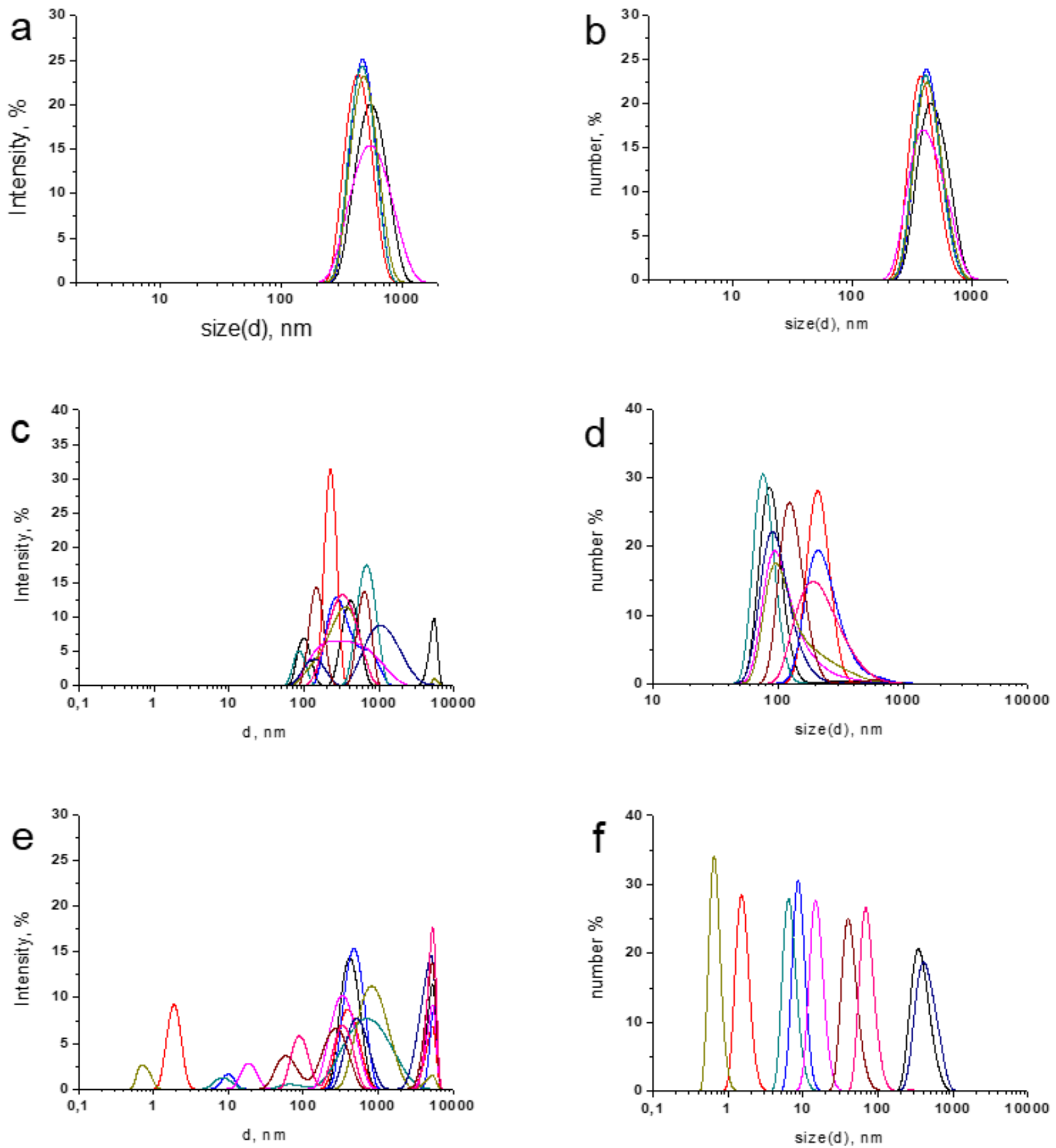

Figure 2. Sequential replicates of DLS particle size distribution by number $(b, d, f)$ and by intensity $(\mathrm{a}, \mathrm{c}, \mathrm{e})$ for tap water sampled in Moscow $(\mathrm{a}, \mathrm{b})$ in San Francisco (c,d) and in Rome $(\mathrm{e}, \mathrm{f})$.

However, a different situation was registered for $\mathrm{KCl}$ solution. In this particular case, no significant differences between distributions by number and by intensity were observed, Figure $1 \mathrm{c}$,d. It means that solid $\mathrm{KCl}$ contains a sufficient amount of microsolid impurities $(100 \mathrm{~nm}<$ size $<1000 \mathrm{~nm})$, and they dominate other fractions, including nanoparticles. The 
latter nevertheless become visible as small nano-shoulders of some bands. Anyhow, the data presented in Figure 1, demonstrate that an undetectable by a particle counter nanofraction may drastically exceed the number of microparticles $\left(\mathrm{HNO}_{3}\right.$ case $)$, or may be comparable with them (case of $\mathrm{KCl}$ in water).

A comparison of tap water samples, taken in different European cities, indicates that the mean microimpurities content is nearly the same. For the particles with size $>100 \mathrm{~nm}$ it varies from 280000 in $1 \mathrm{ml}$ (Amsterdam) to 440000 (Rome). This difference slightly exceeds the experimental error of $20 \%$. A random sample set, presented in Table 4, does not claim to be complete or universal, but it indicates that similar solid background impurities might happen in any laboratory worldwide that uses tap water distillation or deionization and a subsequent preparation of laboratory solutions.

As far as suspended particles concentration is high, DLS reveals a well reproducible particle size distribution. Herewith, both modes (by number and by intensity) match well each other. Some examples are presented in Figure 2. Notably, a relative content of fine nanofractions and microfractions may vary significantly in different cities. In Moscow tap water the microparticles are dominating, Figure $2 a, b$, while in the corresponding sample from Rome (Figure 2e,f) the nano-fraction prevails. Meanwhile, in water from San Francisco the nano- and micro-fractions are comparable with each other.

\subsection{Solid phase characterization by SEM-EDS and XRD}

In order to put some light on the suspended matter nature, the SEM-EDS and XRD analysis of water sample sediments, collected at filters, was performed. We realize that such analysis is partial and not a comprehensive one, because nanosolids are not retarded by $200 \mathrm{~nm}$ filters, commonly used in water treatment studies [9, 12, 13, 17, 29-33]. Even though, we find this information valuable.

SEM images of virgin filter and of a treated filter are presented in Figure 3. It can be seen that after $100 \mathrm{ml}$ DI water filtration, the corresponding image (Figure 3b,c) reveals some solid particles of sub-micrometer size, although some of them are larger than $1 \mu \mathrm{m}$.
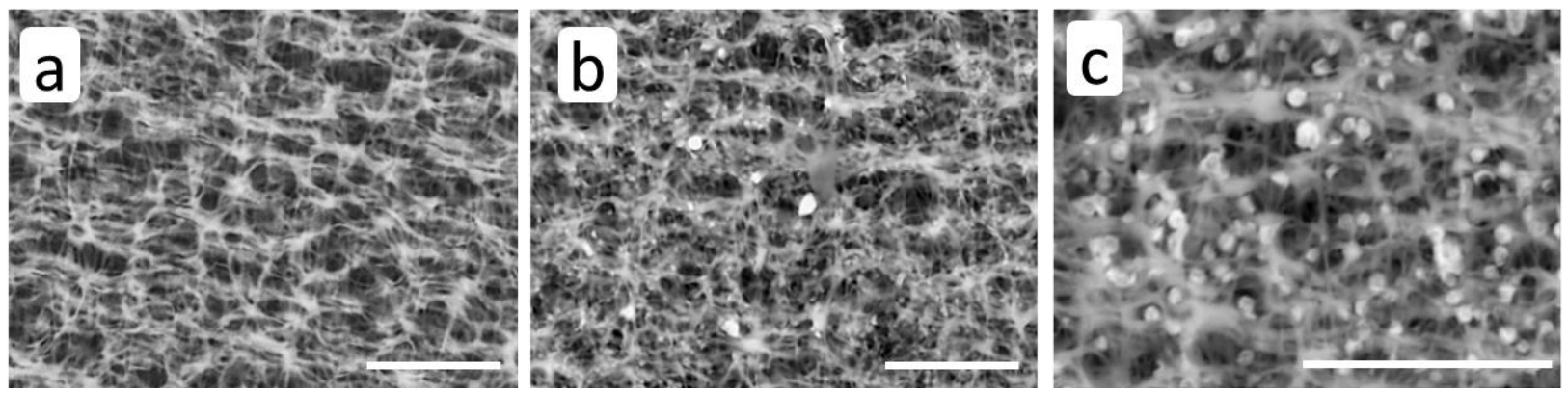

Figure 3. SEM images of PTFE filter surface: virgin sample (a), the same sample after $100 \mathrm{ml}$ distilled water filtration $(\mathrm{b}, \mathrm{c})$. Scale bar corresponds to $10 \mu \mathrm{m}$. 
Meanwhile, the top layer membrane pores are as large as $6 \mu \mathrm{m}$, and some sediment particles below $0.5 \mu \mathrm{m}$ size are retarded by the inner membrane layers. This can be seen in Figure 3c. Thus, some of them are not accounted by EDS analysis, Figure 4.

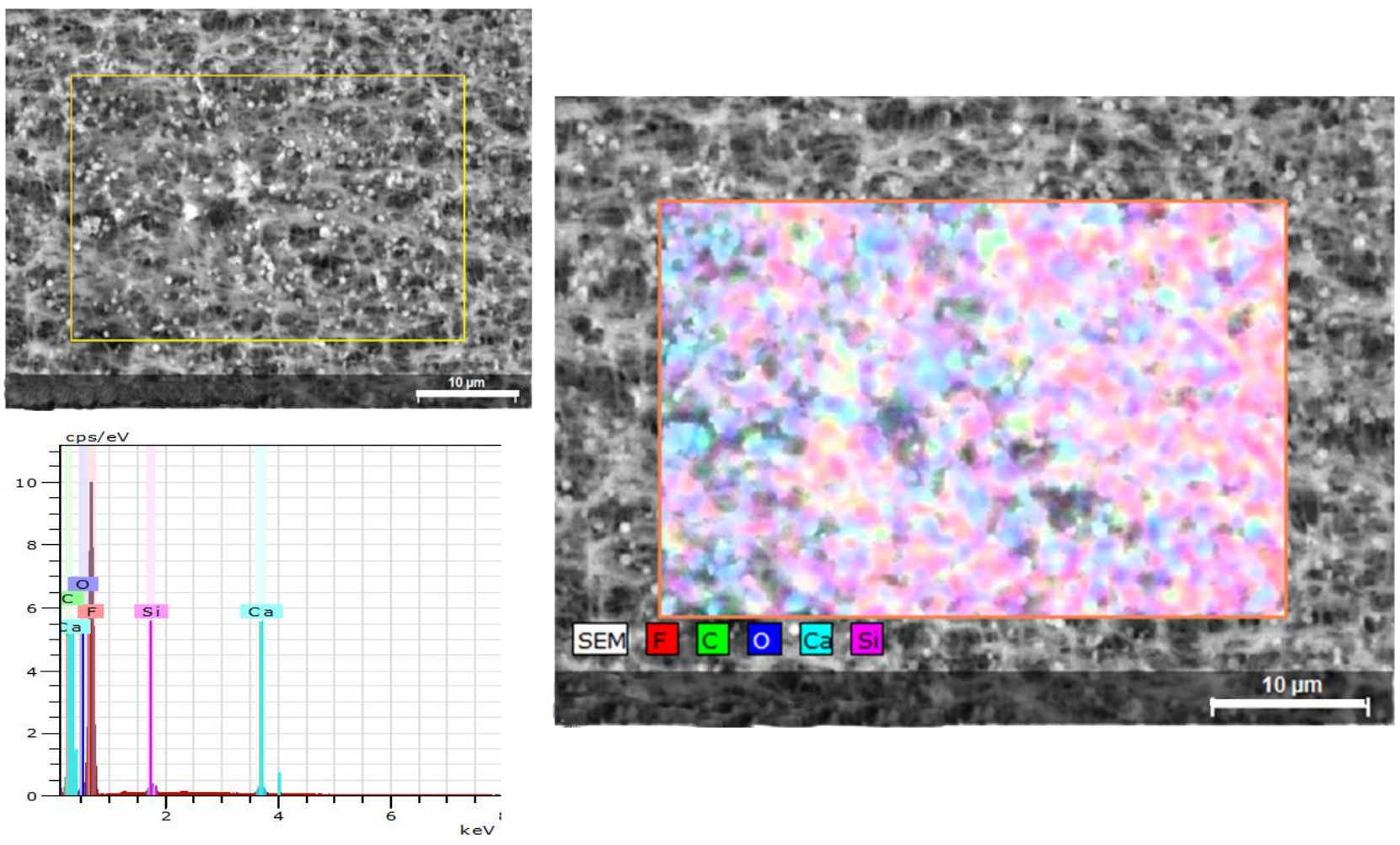

Figure 4. A map of element distribution: SEM-EDS images of PTFE filter surface after $100 \mathrm{ml}$ of distilled water filtration.

The energy-dispersive analysis (EDS) study of the virgin filter surface exhibited only membrane material elements presence: $\mathrm{F}$ (65 at.\%) and C (35 at.\%). The same surface after $100 \mathrm{ml}$ of DI water permeation demonstrated besides $\mathrm{F}$ and $\mathrm{C}$ also $\mathrm{Ca}(0.8$ at.\%), $\mathrm{Si}$ (0.6 at.\%) and $\mathrm{O}(4.3$ at.\%), Figure 5. Notably, the at.\% ratio of $\mathrm{F} / \mathrm{C}$ decreases from 1.86 to 1.76. This was assigned to the increase of carbon content due to some calcium carbonate deposition. At the same time, some Si was found on membrane surface. This indicates presence of either $\mathrm{SiO}_{2}$ or of some silicate solid impurities. It should be noted, that the mapping of individual elements, Figure 5, reveals a different distribution of $\mathrm{Ca}$ and $\mathrm{Si}$, e.g. these elements are concentrated in different particles. This fact reveals clearly that the suspended solid matter is not uniform, but represents a mixture of chemically different particles. Tentatively these are $\mathrm{CaCO}_{3}$ and $\mathrm{SiO}_{2}$.

Figure 5 demonstrates also that both these compounds have different mean size. Evidently, $\mathrm{CaCO}_{3}$ is represented by the larger particles, while $\mathrm{SiO}_{2}$ by the smaller ones. Meanwhile, $\mathrm{Si}$ is distributed over membrane filter surface more uniformly than $\mathrm{Ca}$, and its locations coincide with the background membrane elements $(\mathrm{F}, \mathrm{C})$ location, $e . g$. the spots of a higher membrane fibers density. 

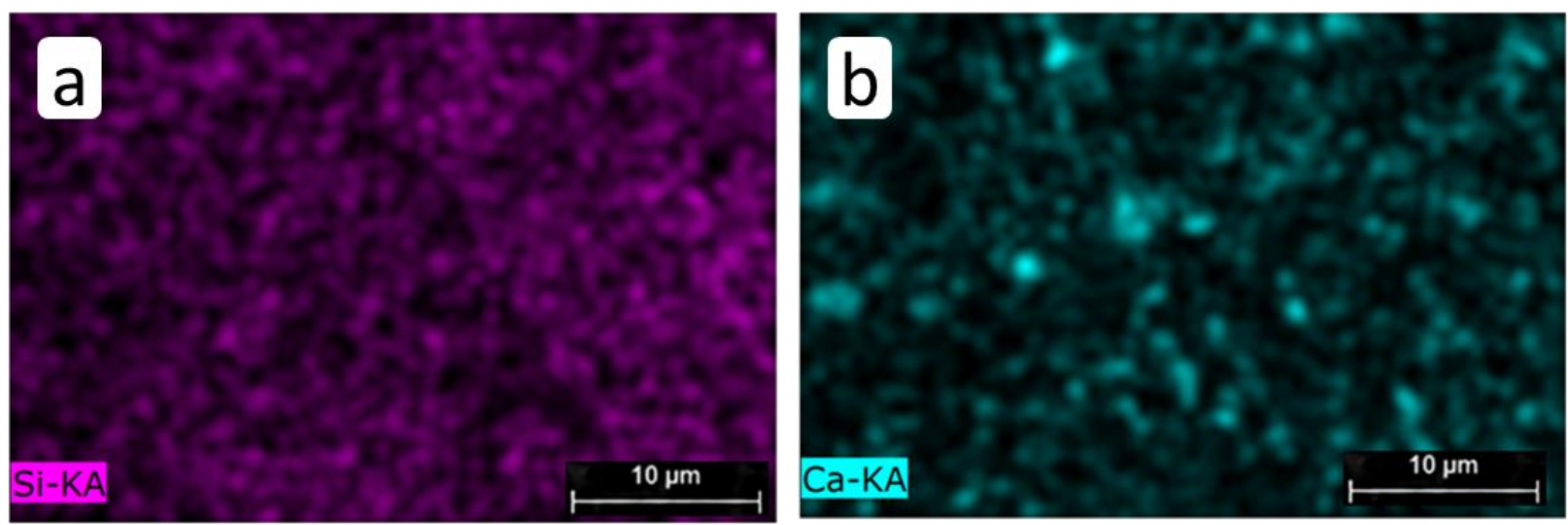

Figure 5. SEM-EDS images of PTFE filter surface showing silica (a) and calcium (b) fouling after $100 \mathrm{ml}$ distilled water filtration.

The lack of $\mathrm{Fe}$ and $\mathrm{Al}$ on membrane surface may be explained by a more fine dispersion of corresponding solids relative to those, represented by $\mathrm{Ca}$ and $\mathrm{Si}$. Tentatively $\mathrm{Fe}$ and $\mathrm{Al}$ bearing particles due to their smaller sizes penetrate deep inside the membrane. Thus, these are retarded by the inner layers of filter and are not detected by the membrane top layer analysis by SEM-EDS technique.

Therefore, the joint ICP, SEM-EDS and particle counter analysis indicate that the solid matter impurities in the distilled water are not uniform by chemical composition and sizes. Tentatively it corresponds to the mixture of $\mathrm{CaCO}_{3}, \mathrm{SiO}_{2}$ and $\mathrm{Fe} / \mathrm{Al}$ hydroxo/oxides with a mean particle size $\mathrm{CaCO}_{3}>\mathrm{SiO}_{2}>\mathrm{Fe} / \mathrm{Al}$ hydroxo/oxides. Notably, this observation refers only to the solid micro-impurities (size $>100 \mathrm{~nm}$ ), while the "nanodust" fraction $(1 \mathrm{~nm}<$ size $<100 \mathrm{~nm})$ still remains a challenge for the recent methods of particle characterization. It is reasonable to suppose, that at least partly its chemical composition is formed by the finest fractions of solid micro-impurities, identified in the present study $\left(\mathrm{CaCO}_{3}, \mathrm{SiO}_{2}\right.$ and $\mathrm{Fe} / \mathrm{Al}$ oxides). However, some other constituents, typical for air pollution: feldspar $\left(\mathrm{K}\left[\mathrm{AlSi}_{3} \mathrm{O}_{8}\right]\right)$, clay minerals (muscovite $\left(\mathrm{KAl}_{2}\left[\mathrm{AlSi}_{3} \mathrm{O}_{10}\right](\mathrm{OH})_{2}\right)$; illite $\left(\mathrm{K}_{0.75}\left(\mathrm{H}_{3} \mathrm{O}\right)_{0.25}\right) \mathrm{Al}_{2}\left(\mathrm{Si}_{3} \mathrm{Al}\right) \mathrm{O}_{10}\left(\left(\mathrm{H}_{2} \mathrm{O}\right)_{0.75}(\mathrm{OH})_{0.25}\right)_{2}$; kaolinite $\left(\mathrm{Al}_{4}\left[\mathrm{Si}_{4} \mathrm{O}_{10}\right](\mathrm{OH})_{8}\right)$, etc. $)$ [7] are also possible.

In any case, recent study indicates, that the listed above solid impurities may and do serve as ideal scale nucleation natural precursors of inorganic scale in a bulk aqueous medium. This was clearly demonstrated by us for barite crystallization [20]. The data on the role of natural solid impurities concentration in gypsum crystallization is currently in press [34] and a paper on further development of our studies of $\mathrm{CaCO}_{3}$ crystallization [35] is in preparation.

\section{Conclusions}

By means of particle counter technique, solid microimpurities were found in all high purity reagents studied, e.g. in distilled and deionized water produced from Moscow tap water, in pure water supplied by Sigma-Aldrich, in nitric acid for semiconductors (Avantor 
Performance Materials Ltd.) and in $\mathrm{KCl}$ (Sigma-Aldrich) 0.1 molar solution in deionized water. The microparticle (size $\geq 100 \mathrm{~nm}$ ) concentration varies from $10^{2}$ to $10^{4}$ units in one $\mathrm{ml}$. At the same time, DLS technique demonstrated, that nanoparticles $(1 \leq$ size $\leq 100 \mathrm{~nm})$ concentration is at least 100-fold higher, than that one of microparticles. All these solid impurities are doomed to become the ideal scale nucleation natural precursors of inorganic scale in a bulk aqueous medium.

A common procedure of dust removal via microfiltration (200 nm filter) is capable to reduce the microparticle concentration, but fails to remove completely even the fractions with sizes above $200 \mathrm{~nm}$.

The chemical composition of solid impurities in distilled and deionized water derived from Moscow municipal tap water includes, but is not reduced entirely to $\mathrm{CaCO}_{3}, \mathrm{SiO}_{2}$ and $\mathrm{Fe} / \mathrm{Al}$ hydroxo/oxides with a mean particle size $\mathrm{CaCO}_{3}>\mathrm{SiO}_{2}>\mathrm{Fe} / \mathrm{Al}$ hydroxo/oxides.

The particle counter analysis of tap water from different cities around the globe indicates that microparticles content is approximately the same for different geographic regions, apparently due to similar water purification techniques and similar internationally accepted standards. Thus, the problem of laboratory chemicals and solvents purification from crystal nucleation natural precursors is also common, although not yet broadly recognized.

\section{Acknowledgments}

The authors would like to thank the Russian Science Foundation, Project No. 19-79-10220, for the financial support of the present study.

\section{References}

1. T.H. Zhang and X.Y. Liu, Nucleation: What Happens at the Initial Stage?, Angew. Chem., 2009, 121, 1334-1338. doi: 10.1002/anie.200804743

2. D. Gebauer, M. Kellermeier, J.D. Gale, L. Bergström and H. Cölfen, Pre-nucleation clusters as solute precursors in crystallization, Chem. Soc. Rev., 2014, 43, 2348-2371. doi: $10.1039 / \mathrm{c} 3 \mathrm{cs} 60451 \mathrm{a}$

3. G.C. Sosso, J. Chen, S.J. Cox, M. Fitzner, P. Pedevilla, A. Zen and A. Michaelides, Crystal nucleation in liquids: Open questions and future challenges in molecular dynamics simulations, Chem. Rev., 2016, 116, 7078-7116. doi: 10.1021/acs.chemrev.5b00744

4. E.A. Abdel-Aal, H.M. Abdel-Ghafar and B.E. El Anadouli, New Findings about Nucleation and Crystal Growth of Reverse Osmosis Desalination Scales with and without Inhibitor, Cryst. Growth Des., 2015, 15, 5133-5137. doi: 10.1021/acs.cgd.5b01091

5. M.N. Elliott, Scale control by threshold treatment, Desalination, 1970, 8, 221-236. doi: 10.1016/S0011-9164(00)80231-3

6. K.G. Cooper, L.G. Hanlon, G.-M., Smart and R.E. Talbot, The threshold scale inhibition phenomenon, Desalination, 1979, 31, 257-266. doi: 10.1016/S0011-9164(00)88526-4 
7. M. Paramonov, R.O. David, R. Kretzschmar and Z.A. Kanji, A laboratory investigation of the ice nucleation efficiency of three types of mineral and soil dust, Atmos. Chem. Phys., 2018, 18, no. 22, 16515-16536. doi: 10.5194/acp-18-16515-2018

8. K. Li, S. Xu, W. Shi, M. He, H. Li, S. Li, X. Zhou, J. Wang and Y. Song, Investigating the effects of solid surfaces on ice nucleation, Langmuir, 2012, 28, no. 29, 10749-10754. doi: $10.1021 / 1 a 3014915$

9. J. Benecke, J. Rozova and M. Ernst, Anti-scale effects of select organic macromolecules on gypsum bulk and surface crystallization during reverse osmosis desalination, Sep. Purif. Technol., 2018, 198, 68-78. doi: 10.1016/j.seppur.2016.11.068

10. Y.M. Al-Roomi and K.F. Hussain, Potential kinetic model for scaling and scale inhibition mechanism, Desalination, 2016, 393, 186-195. doi: 10.1016/j.desal.2015.07.025

11. O.D. Linnikov, Mechanism of precipitate formation during spontaneous crystallization from supersaturated aqueous solutions, Russ. Chem. Rev., 2014, 83, no. 4, 343-364. doi: 10.1070/RC2014v083n04ABEH004399

12. A. Lancia, D. Musmarra and M. Prisciandaro, Measuring induction period for calcium sulfate dehydrate precipitation, AICHE J., 1999, 45, 390-397. doi: 10.1002/aic.690450218

13. M. Prisciandaro, A. Lancia and D. Musmarra, Gypsum nucleation into sodium chloride solutions, AICHE J., 2001, 47, 929-934. doi: 10.1002/aic.690470415

14. W. Yu, D. Song, W. Chen and H. Yang, Antiscalants in RO membrane scaling control, Water Res., 2020, 183, 115985. doi: 10.1016/j.watres.2020.115985

15. W. Ying, N. Siebdrath, W. Uhl, V. Gitis and M. Herzberg, New insights on early stages of RO membranes fouling during tertiary wastewater desalination, J. Membr. Sci., 2014, 466, 26-35. doi: 10.1016/j.memsci.2014.04.027

16. A.E.S. Van Driessche, T.M. Stawski and M. Kellermeier, Calcium sulfate precipitation pathways in natural and engineered environments, Chem. Geol., 2019, 530, 119274. doi: 10.1016/j.chemgeo.2019.119274

17. T.M. Stawski, A.E.S. Van Driessche, M. Ossorio, J.D. Rodriguez-Blanco, R. Besselink and L.G. Benning, Formation of calcium sulfate through the aggregation of sub-3 nanometre primary species, Nat. Commun., 2016, 7, 11177. doi: 10.1038/ncomms11177

18. S.L. Gromov, Deposit formation in spiral-wound reverse osmosis and nanofiltration elements and ways of preventing it, Therm. Eng., 2014, 61, no. 6, 433-441. doi: 10.1134/S0040601514060032

19. M. Oshchepkov, V. Golovesov, A. Ryabova, A. Redchuk, S. Tkachenko, A. Pervov and K. Popov, Gypsum crystallization during reverse osmosis desalination of water with high sulfate content in presence of a novel fluorescent-tagged polyacrylate, Crystals, 2020, 10, 309. doi: $\underline{10.3390 / \text { cryst10040309 }}$ 
20. M. Oshchepkov, K. Popov, A. Ryabova, A. Redchuk, S. Tkachenko, J. Dikareva and E. Koltinova, Barite crystallization in presence of novel fluorescent-tagged antiscalants, Int. J. Corros. Scale Inhib., 2019, 8, no. 4, 998-1021. doi: 10.17675/2305-6894-2019$\underline{8-4-12}$

21. M. Oshchepkov, S. Kamagurov, S. Tkachenko, A. Ryabova and K. Popov, An Insight into the Mechanisms of the scale inhibition. A case study of a novel task-specific fluorescent-tagged scale Inhibitor location on gypsum crystals, ChemNanoMat, 2019, 5, 586-592. doi: $10.1002 / \mathrm{cnma.201800660}$

22. A.G. Dikundwar, S. Pal, P. Chodon, R. Narasimhamurthy, P. Kameshwar, M. Sundaram and H. Bhutani, Solid state behavior of impurities during "In-Process" phase purity analysis of an AP, Org. Process Res. Dev., 2019, 23, no. 2, 269-273. doi: 10.1021/acs.oprd.8b00334

23. L. Owen and M. Tragesser, Semiconductors. Fundamentals of high-purity water used in microelectronic applications, Ultrapure Water, 2001, 18, no. 5, 41, 43-46, 48.

24. D. Monticelli, A. Castelletti, D. Citati, S. Recchia and C. Dossi, How to efficiently produce ultrapure acids, Int. J. Anal. Chem., 2019, 1, 5180610. doi: $10.1155 / 2019 / 5180610$

25. Sigma-Aldrich Catalogue, https://www.sigmaaldrich.com

26. Nanocount UPW Liquid Particle Counter. NanoCount 30. http://www.golighthouse.com

27. International standard ISO 13321 Methods for determination of particle size distribution. Part 8: Photon correlation spectroscopy, International Organization for Standartisation (ISO), 1996.

28. Measurement of suspended particles by quasi-elastic light scattering, Ed.: B.E. Dahneke, Wiley, 1983.

29. S. He, J.E. Oddo and M.B. Tomson, The nucleation kinetics of calcium sulfate dehydrate in $\mathrm{NaCl}$ solution up to $6 \mathrm{~m}$ and $90^{\circ} \mathrm{C}, \mathrm{J}$. Colloid Interface Sci., 1994, 162, 297-303. doi: $\underline{10.1006 / j \text { jis.1994.1042 }}$

30. F. Alimi, H. Elfil and A. Gadri, Kinetics of the precipitation of calcium sulfate dihydrate in a desalination unit, Desalination, 2003, 57, 9-16. doi: 10.1016/S00119164(03)00426-0

31. L. Nicoleau, A.E.S. Van Driessche and M. Kellermeier, Kinetic analysis of the role of polymers in mineral nucleation. The example of gypsum, Cem. Concr. Res., 2019, 124, 105837. doi: 10.1016/j.cemconres.2019.105837

32. A.E.S. Van Driessche, J.M. García-Ruiz, J.M. Delgado-López and G. Sazaki, In Situ observation of step dynamics on gypsum crystals, Cryst. Growth Des., 2010, 10, 39093916. doi: $10.1021 / \mathrm{cg} 100323 \mathrm{e}$

33. T.M. Stawski, A.E.S. Van Driessche, R. Besselink, E.H. Byrne, P. Raiteri, J.D. Gale and L.G. Benning, The structure of $\mathrm{CaSO}_{4}$ nanorods: the precursor of gypsum, J. Phys. Chem., 2019, 123, 23151-23158. doi: 10.1021/acs.jpcc.9b04268 
34. M. Oshchepkov, K. Popov, A. Kovalenko, A. Redchuk, J. Dikareva and I. Pochitalkina, Initial stages of gypsum nucleation: the role of "nano/microdust", Minerals, 2020, in press.

35. I.A. Pochitalkina, P.A. Kekin, A.N. Morozov, I.A. Petropavlovskii and D.F. Kondakov, Crystallization Kinetics of Calcium Carbonate at a Stoichiometric Ratio of Components, Russ. J. Phys. Chem. A, 2016, 90, 2346-2351. doi: 10.1134/S0036024416120232 